\title{
Long-term survival of structurally compromised tooth preserved with crown lengthening procedure and restorative treatment: A pilot retrospective analysis
}

\author{
Sajjad Ashnagar (iD) | Shayan Barootchi (D) | Andrea Ravidá (D) | Mustafa Tattan (iD | \\ Hom-Lay Wang (i) | Chin-Wei Wang (i)
}

Department of Periodontics and Oral Medicine, University of Michigan, School of Dentistry, Ann Arbor, Michigan

\section{Correspondence}

Chin-Wei Wang, Department of Periodontics and Oral Medicine, University of Michigan School of Dentistry, $1011 \mathrm{~N}$. University Ave., Ann Arbor, MI 48109-1078. Email: jeffwa@umich.edu

Funding information

This study was funded by Rackham Graduate School, University of Michigan, Ann Arbor, Michigan.

\begin{abstract}
Aim: Since there is limited study to assist in an evidenced-based decision whether to extract or preserve a structurally compromised tooth, the aim of this retrospective study was to investigate the long-term survival rate of tooth preservation after crown lengthening procedure (CLP) and restorative treatments.

Methods: Electronic and paper chart of patients received CLP in our graduate clinic from 1990 to 2015 were reviewed. Statistical analysis was done using Cox regression analysis and Kaplan-Meier estimator.

Results: A total of 766 coded charts were initially collected. Four hundred and fourteen cases were included in the final analysis. The Kaplan-Meier estimate shows a cumulative survival rate of $88.3 \%$ in 5 years, $78.4 \%$ in 10 years and $68.1 \%$ in 15 years. In terms of reasons for failure, restorative problem such as recurrent decays was the main issue (35.2\%), followed by fracture (29.6\%), endodontic complications (23.9\%) and periodontal breakdown (11.3\%).

Conclusions: Within the limitation of this study, structurally compromised teeth have a reasonable long-term survival rate close to $80 \%$ after 10 years in a teaching institute. Patients with high fracture or caries risk may pose a higher chance of failure. Objective information should be presented to the patient to arrive at an evidencebased decision.
\end{abstract}

\section{KEYWORDS}

crown lengthening, periodontics, prognosis, survival rate, tooth preparation

\section{1 | INTRODUCTION}

Teeth with extensive deep caries, crown fracture, short clinical crown and severe attrition may end up with insufficient tooth structure to support or retain a quality restoration (Hempton \& Dominici, 2010; Palomo \& Kopczyk, 1978). Additionally, deep subgingivally prepared tooth margins have a higher chance of creating ill-fitted restorations that may violate supracrestal attachment and compromise periodontal health (Padbury, Eber, \& Wang, 2003; Palomo \&
Kopczyk, 1978; Pilalas, Tsalikis, \& Tatakis, 2016). In these situations, a crown lengthening procedure (CLP) with osseous recontouring can assist in re-establishing supracrestal tissue (Carnevale, Sterrantino, $\&$ Di, 1983; Oakley et al., 1999) and exposing a greater amount of tooth structure to better support future restorations (Allen, 1993).

With the recent popularity of dental implant therapy, there is a growing tendency among the dental community towards replacing structurally compromised teeth with implants when treatment planning. This may be due to various reasons, including questionable 
restorability of the tooth, level of the bone, cost-benefit ratio and patient preference (Iqbal \& Kim, 2008). The clinicians' interpretation and preference also play a major role in communication with the patients. Despite the presence of a reasonable rational behind many tooth extractions, there is an inconsistency regarding the criteria of a hopeless or poor prognosis for a tooth (Lundgren, Rylander, \& Laurell, 2008). Although many studies, including systematic reviews, have demonstrated comparable survival of endodontically treated teeth with dental implants (Torabinejad \& Goodacre, 2006), some clinicians may still consider dental implants over compromised tooth needing multi-interdisciplinary care (Giannobile \& Lang, 2016; Packer, 2007; Stockhausen, Aseltine, Matthews, \& Kaufman, 2011). Patients rely on the information received from their dental care providers during decision-making. This information might be solely based on their presumptions and preferences without objective and evidence-based information regarding the tooth's prognosis, especially for structurally compromised dentition.

Limited evidence is available in assessing the long-term outcomes of teeth preserved after CLP, as most studies only have short-term results focusing on the tissue level changes, with small number of patients, or restricted to endodontically treated teeth (Moghaddam, Radafshar, Taramsari, \& Darabi, 2014; Patil, Kulkarni, Thakur, \& Naik, 2016). Given the limited information to provide evidence-based decisions, the primary aim of this retrospective study was to determine the long-term overall survival rate of structurally compromised teeth that underwent CLP and restorative treatment. Their reasons for failure were also investigated to assist both patients and clinicians for decision-making.

\section{MATERIAL AND METHODS}

\section{1 | Study design}

The present study was conducted according to the principles embodied in the Helsinki Declaration of 1975, as revised in 2000 for biomedical research involving human subjects and was approved by the Institutional Review Board for Human Studies, School of Dentistry, University of Michigan, Ann Arbor, MI, USA (HUM00131960) to be conducted at the Department of Periodontics and Oral Medicine within the same institution.

In this retrospective study, all the clinical records of patients who underwent a CLP at the University of Michigan School of Dentistry (UMSOD) teaching clinic between January 1990 and January 2015 were obtained. All paper and digital files of the patients for possible inclusion were scanned and analysed by four authors (SA, AR, SB and MT). During each stage in case of any disagreements, another author with expertise in the matter (CJW) was referred to in pursuit of a resolution.

\section{2 | Eligibility criteria}

Patients were included in the study if they underwent functional CLP at the UMSOD. Subsequently any of the following reasons resulted

\section{Clinical Relevance}

Scientific rationale for the study: There is limited study assessing the long-term prognosis of structurally compromised teeth requiring crown lengthening procedure (CLP) to assist in an evidence-based decision whether to extract the tooth.

Principal Findings: Results of the current study suggest a reasonable 10 -year survival rate of close to $80 \%$ after treatment at a teaching institute. Secondary decays or tooth fracture was the most common cause of failure.

Practical implications: Structurally compromised tooth can be preserved with CLP and restoration for a reasonable long-term survival rate. Objective information should be presented to patients to make a well-informed decision.

in the exclusion of that patient or file from the study: (a) no follow-up visits or less than 1-year follow-up; (b) incomplete charts or incorrect entry of codes; (c) destroyed records or inaccessible files; (d) erroneous or change of the treatment plan; (e) patients receiving a CLP solely for aesthetic purposes (non-functional or without bone removal).

\section{3 | Data collections}

Within the review period, a thorough screening of all CLP-treated teeth at the UMSOD was carried out in the specified timeframe for assessment and possible inclusion. All relevant clinical data were gathered from the files regarding tooth number, survival time of the tooth (in months) and cause of extraction in the case of a failure.

Crown lengthening procedure survival was defined as any tooth that had previously undergone a functional CLP and remained in function, within the patient's persisting dentition, until the last given evidence of the follow-up period. Contrarily, CLP failure was defined as any tooth that, following a previously performed functional CLP and delivered restoration, had been extracted due to any given reason along the observation period.

The survival time was calculated from the day of the surgical procedure (baseline) to the most recent visit with evidence showing that the tooth had survived.

\section{4 | Statistical analysis}

Survival analysis was performed according to the Kaplan-Meier estimator to obtain the cumulative survival rates at different time points. All statistical analyses were performed using statistical software for Macintosh ( $\mathrm{R}$ studio, $\mathrm{R}$ studio, Inc.). The survival plot was created based on Cox regression analysis with their estimated confidence interval $(\mathrm{Cl})$. 


\section{3 | RESULTS}

A total of 766 individuals' CLP-treated teeth that were initially indicated were thoroughly reviewed and screened by three examiners (SA, AR and SB). After careful evaluation against the aforementioned exclusion criteria, a total number of 414 teeth (from 358 patients) were included.

In summary, the number of the excluded files is as follows: (a) lack of follow-up (127), (b) insufficient (<1 year) follow-up recall (80), (c) inaccessible files or destroyed records (50), (d) aesthetic CLP (148), (e) incomplete data on file (30), and (f) erroneous or incomplete treatment planning (11). Regarding the main reason for performing CLP, out of 414 teeth, 151 were carried out due to subgingival caries, 144 due to creation of the ferrule effect for crown placement, 111 to re-establish biologic width and eight cases were for unidentified reasons. Surgical procedures were performed by graduate level clinical residents at our teaching clinic. Restoration and prosthetic work was done by both graduate level residents and dental students. The demographic information, total chart review workflow and exclusion process are presented in Figure 1.

From the total 414 teeth included and followed, 71 cases of CLP were considered as failure and were subsequently extracted. Of the 258 teeth with RCT, crown and CLP, 53 teeth were failed in the followup period. The average follow-up for all the cases was 85.5 months (range: 12-257 months). Eighty-seven out of 414 included cases showed a history of periodontitis after treatment in our included data. Out of 71 failures, 34 of failures showed history of periodontitis. However, periodontal breakdown was the cause of extraction in only four of these 34 cases. Most cases with long-term follow-ups had their recalls and maintenance within the institute. The overall estimated cumulative survival rate based on the Kaplan-Meier analysis showed the rate of $88.3 \%$ at 5 years, $78.4 \%$ at 10 years and $68.1 \%$ at 15 years. The survival plot and bar graphs are shown in Figure 2a,b.

The survival rate based on different tooth types and locations and number of failures are presented in Table 1 and Figure 3. In general, maxillary teeth had slightly higher failure rate compared to the mandibular teeth ( $18 \%$ vs. $16.2 \%$ ). While mandibular incisors presented with the highest cumulative survival rate (100\%), only four teeth were included. Both maxillary and mandibular premolar teeth have highest failure rate close to $20 \%$.
The causes of tooth failure and subsequent extraction were categorized as the following: (a) restorative reasons (majority are secondary caries or repeated dislodgement of crowns) accounting for $35.2 \%$ of total failures, (b) tooth fracture, consisting of $29.6 \%$ failures, (c) endodontic reasons (presence of apical radiolucency on radiograph, a sinus tract, drainage or such compilatory factors, while re-treatment not possible), accounting for $23.9 \%$ of failures and lastly, (d) periodontal origin (extraction due to severe loss of the supporting periodontium, severe furcation involvement or mobility) accounting for $11.3 \%$ of failures (Table 2, Figure 4). Fracture is the leading cause of failure in 5 - to 10 -year period by accounting for $36 \%$ of the total failures; while in 10-15 years, caries/restorative reasons are lead reasons of failure consisting of $50 \%$ failed cases. Of note, out of 33 cases, which were followed up for more than 15 years (up to 21.4 years), only one case failed.

\section{4 | DISCUSSION}

There are limited studies in the literature to assess the long-term survival of teeth preserved with CLP. While making a decision whether to extract or preserve a structurally compromised tooth, patients oftentimes do not receive enough objective information to make a fair judgement. Clinicians may also have their own perception regarding the longevity of tooth preservation after CLP, which may result from the popularity of dental implants in general practices. The advantage of CLP with the restorative treatment is to preserve the tooth and reserve dental implant as a latter option if needed; one of the disadvantages of having CLP to preserve the tooth may be multiple treatment procedures involving interdisciplinary care. The impression that implant therapy is more predictable than preserving tooth may be true in the short term but the emerging concern about the dental implant complications should also be considered. Since there is limited study investigated the long-term survival rate of CLP, the prognosis of structurally compromised tooth preserved after CLP needs to be clarified.

In this study, it was found that teeth preserved after CLP have a survival rate of $88.3 \%$ in 5 years, $78.4 \%$ in 10 years and $68.1 \%$ in 15 years. These data are derived from a teaching institute where CLPs, root canal treatment and restorations were all done by
FIGURE 1 Total chart review workflow and exclusion criteria

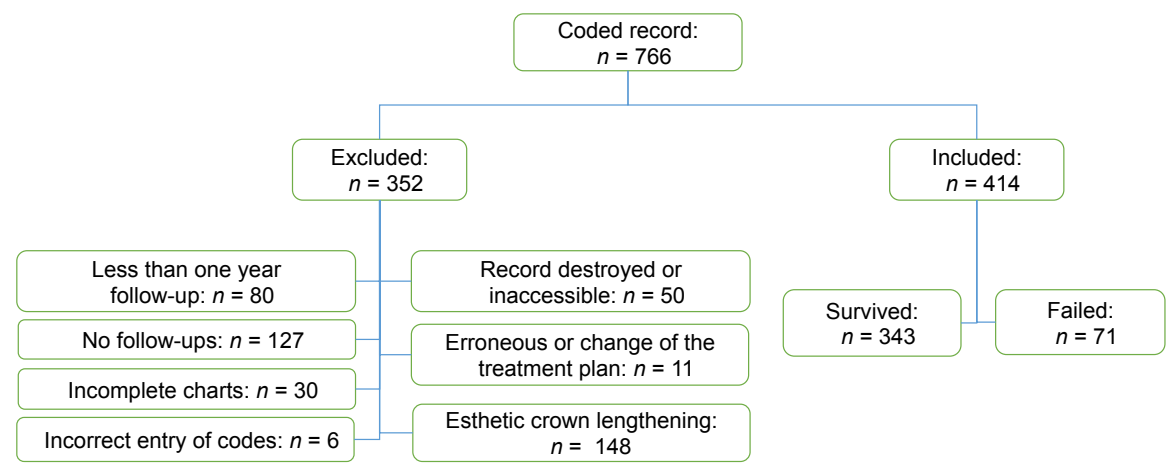




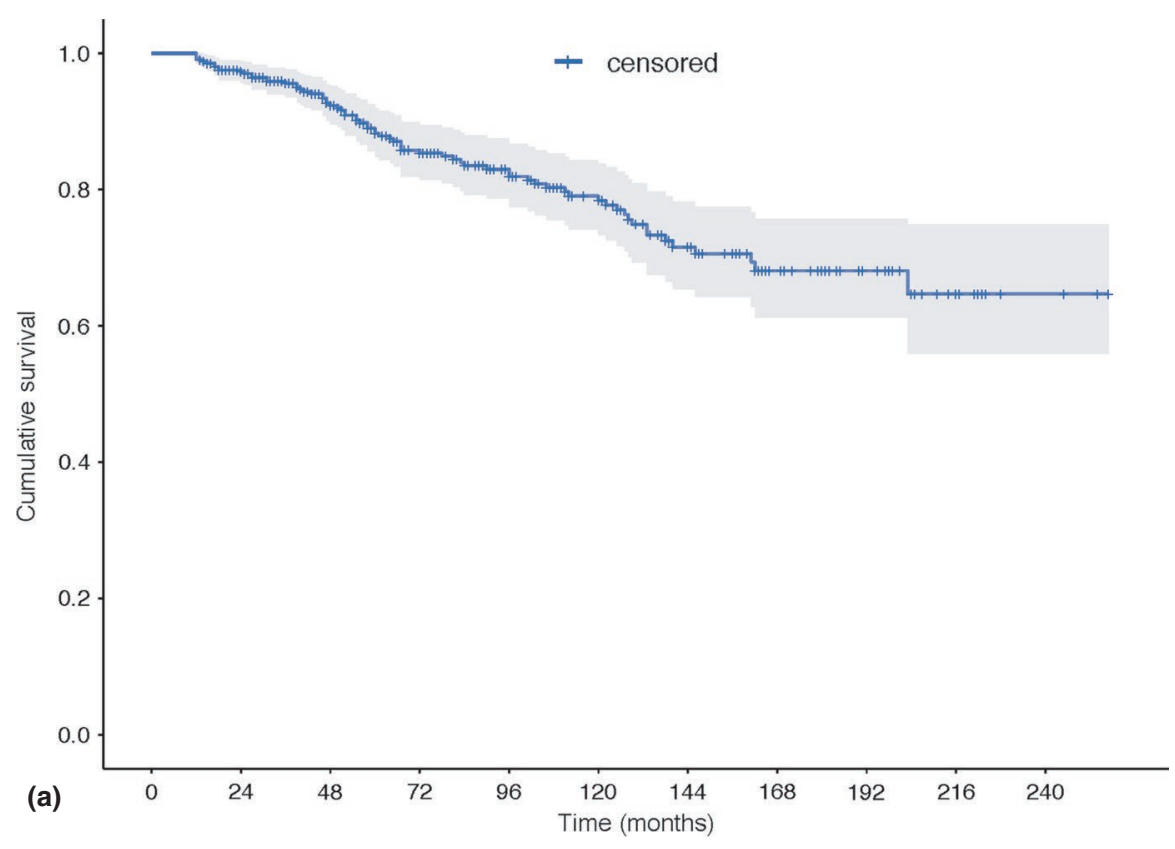

FIGURE 2 Survival rate of structurally compromised teeth preserved with the treatment and crown lengthening procedure. a. Survival plot based on Cox regression analysis, b. Cumulative survival at 5-year intervals using Kaplan-Meier analysis

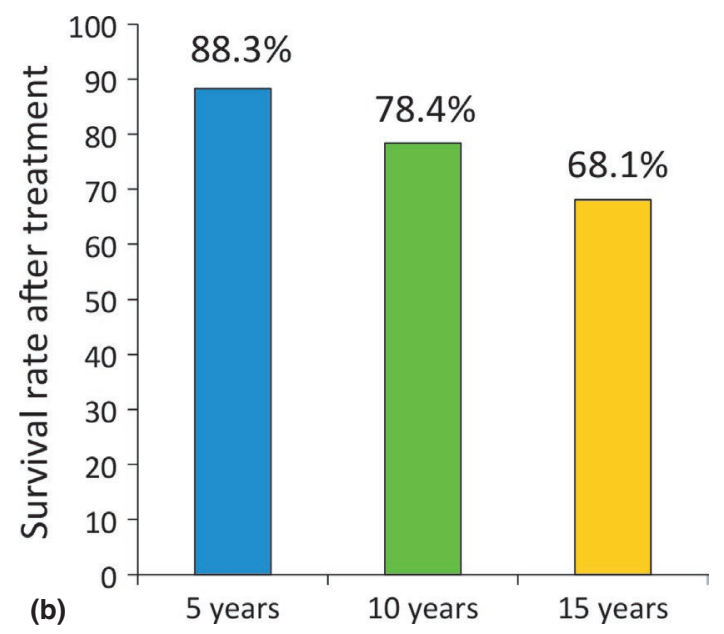

graduate residents and predoctoral students. Although all the procedures were supervised by appointed faculties, the learning curve of the students and patient factors should be noted. The survival rate should be higher if the treatments were done by the experienced clinicians and specialists with well-coordinated plans. Another factor that might lower the long-term survival rate is the statistical method with Kaplan-Meier survival analysis since it assumes the subjects who lost follow-up (censored) have the same survival rate compared to the subjects that remain in the study. We have more than half of the subjects lost follow-up after 10 years and that may have a major impact on the survival rate after 15 years. Of note, out of 35 teeth followed for more than 15 years, only one tooth failed.

Patil et al. (2016) studied the outcome of CLP retrospectively with an average 4.2 years and found $100 \%$ survival rate of teeth treated with CLP. They reported no furcation involvement in teeth with CLP. However, their sample size was very low for about 25 teeth. Dibart, Capri, Kachouh, Dyke, \& Nunn (2003) also reported 100\% survival rate after 5 years with 26 teeth, but furcation involvement occurred in $38.5 \%$ of molars undergone CLP. However, there's no radiographic evaluation right after CLP. The radiolucency seen in furcation after 5 years might be due to ostectomy and osseous contouring during the procedure, not pathologic bone loss. The limitation of the current study is the lack of consistent information in the chart review to determine the "success" of the case during follow-up visits but most cases present with satisfactory outcome and healthy periodontal conditions for function and maintenance. The most comparable study is Moghaddam et al. (2014) which reported a survival rate of $96 \%$ in 5 years and $83.1 \%$ in 10 years. They included 245 cases needing all three disciplines of root canal treatment, CLP and crown (with or without post). This inclusion criterion makes the application only limited to the teeth that required root canal treatment and crowns. In the present study, only $62 \%$ ( 258 out of 414 ) of included cases had all three discipline of treatment. The success rate was reported higher compared to our study. This may be attributed to clinician experience, endodontic treatment and the crown delivery to avoid tooth fracture. 
TAB LE 1 Demographic data and failure rate according to the tooth location

\begin{tabular}{|llll|}
\hline & $\begin{array}{l}\text { Patient } \\
\text { no. }\end{array}$ & $\begin{array}{l}\text { Mean } \\
\text { age }\end{array}$ & $\begin{array}{l}\text { No. Male/ } \\
\text { Female }\end{array}$ \\
\hline Demographic & 358 & 54.0 & $183 / 175$ \\
\hline Total no. & $\begin{array}{l}\text { Failed } \\
\text { no. }\end{array}$ & $\begin{array}{l}\text { Failure } \\
\text { rate (\%) }\end{array}$ \\
\hline Maxillary molars & 86 & 15 & 17.4 \\
\hline Maxillary premolars & 69 & 14 & 20.3 \\
\hline Maxillary canines & 31 & 5 & 16.1 \\
\hline Maxillary incisors & 25 & 4 & 16 \\
\hline Mandibular molars & 139 & 22 & 15.8 \\
\hline Mandibular premolars & 50 & 10 & 20 \\
\hline Mandibular canines & 10 & 1 & 10 \\
\hline Mandibular incisors & 4 & 0 & 0 \\
\hline Total & 414 & 71 & 100 \\
\hline Maxillary teeth & 211 & 38 & 18 \\
\hline Mandibular teeth & 203 & 33 & 16.2 \\
\hline
\end{tabular}

After reviewing reasons for the failure of CLP in this study, it was found that restorative problems, such as recurrent decays or repeated dislodgement of the crowns, constitute the major reason (35.2\%) for failure, followed by fracture (29.6\%). Endodontic failure and periodontal involvement were recorded in $23.9 \%$ and $11.3 \%$ of the cases, respectively. This is in line with the results of Moghaddam et al. (2014) study, which reported restorative problem (secondary caries) being the most common cause of failure (5 out of 18, 27.8\%). Second common cause of failure was vertical root fracture with $22.2 \%$ contribution. Other nine cases (50\%) were not identified in detailed, but mentioned for endodontic problem, furcation involvement and other reasons. In addition, in patients who had failed tooth preserved after CLP and restorations, we also analysed the survival of rate of other teeth without CLP but only crown and endodontic treatment were done. A total of 109 teeth were included in the analysis with Kaplan-Meier survival analysis. For other teeth that had crown placed, 5-year survival rate was $92.6 \%$ and the 10 -year survival was 70.6\%; whereas with endodontic treatment, the 5 -year survival rate was estimated as $82 \%$, and 10 -year survival is $66.8 \%$. The teeth with both treatments yielded a 5 -year survival rate of $88.7 \%$, and a 10 year survival of $69.3 \%$. Patients who lost their teeth after CLP and restorative treatment seem to have a much lower baseline survival rate for other teeth without CLP. This may imply prognostic factors at a subject level, as the top two reasons for failure (recurrent caries risk and fracture risk) can be assessed at the subject level as well.

Therefore, high caries or fracture risk patients may pose higher risk of failure or re-treatment, which should be taken into consideration when assessing the prognosis for the tooth. One study proposed a dmft score of 5 or higher as a predictor for caries risk, which can be implemented during treatment planning (Motohashi et al., 2006). Also, it has been reported that the presence of fracture lines in the enamel increases the relative size of the restoration, and the loss of dentinal support is associated with a higher fracture risk (Bader, Shugars, \& Martin, 2004). This emphasizes the importance of maintenance care regarding the patient oral hygiene and use of fluoridated toothpaste and constant monitoring about the occlusion and risk of tooth fracturing.

Interestingly, fracture rate increased during 5-10 years (36\%) while recurrent decays occur more frequently after 10 years ( 50\%). Endodontic complications and periodontal involvement are relatively consistent during the observational periods. It was also noted that maxillary teeth have slightly higher failure rate after CLP compared to their corresponding tooth type in mandible. Several hypotheses can be proposed for this correlation, namely more complex furcation in molars, root concavity, less saliva cleansing environment and nonaxial forces during mastication for front teeth in the maxillae compared to mandible (Torbjörner \& Fransson, 2004). Overall, maxillary premolars have highest failure rate and mandibular incisors have the lowest yet very few teeth were included. Of note, all the teeth with complications were extracted and no attempts were made for further management. Except for root fracture, some of the minor complications may be able to resolve and still preserve the tooth. However, it is likely that most clinicians and patients would not opt for another round of treatments.

In order to increase the success and survival rate of the tooth preserved, thorough pre-operative assessment, including restorability, endodontic and periodontal condition, should be performed to reduce complications. Well-coordinated treatment and quality interdisciplinary care are also important to achieve the ideal results and long-term success. Minimally invasive approach may be one of the keys to preserve as much tooth structure as possible to avoid tooth fracture, including conservative tooth preparations, minimizing canal debridement and coronal access opening if root canal treatment is indicated (Krishan et al., 2014). Further studies on the prognostic factors for CLP are warranted to provide more precise assessment and management of the given tooth.

When deciding whether to preserve a structurally compromised tooth with CLP and restoration or replacing it with a dental implant,

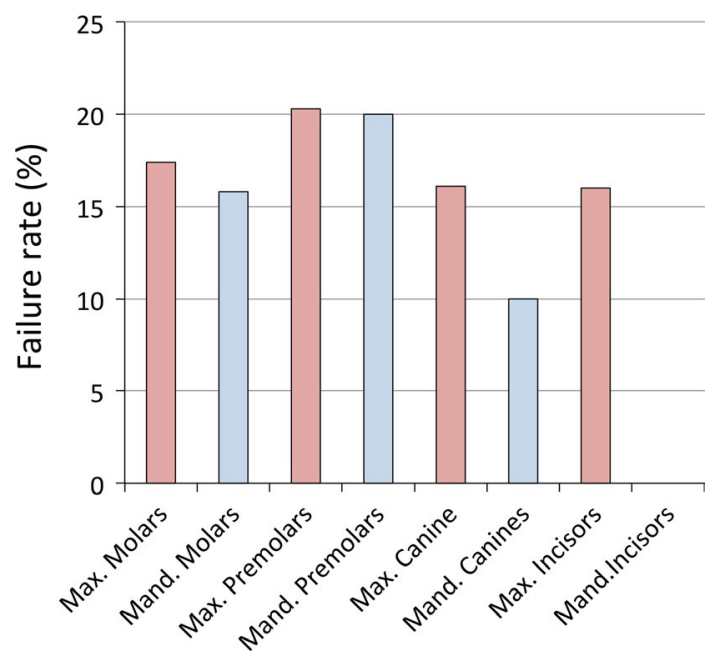

FIGURE 3 Failure rate based on tooth locations 


\begin{tabular}{|c|c|c|c|c|c|c|c|c|c|c|}
\hline \multirow{2}{*}{$\begin{array}{l}\text { Time } \\
\text { Average (months) } \\
\text { Reason }\end{array}$} & \multicolumn{2}{|c|}{$\begin{array}{l}1-5 \text { years } \\
34.9\end{array}$} & \multicolumn{2}{|c|}{$\begin{array}{l}5-10 \text { years } \\
85.5\end{array}$} & \multicolumn{2}{|c|}{$\begin{array}{l}10-15 \text { years } \\
145.2\end{array}$} & \multicolumn{2}{|c|}{$\begin{array}{l}15+\text { years } \\
206.9\end{array}$} & \multicolumn{2}{|c|}{$\begin{array}{l}\text { Overall } \\
85.5\end{array}$} \\
\hline & $n$ & $\%$ & $n$ & $\%$ & $n$ & $\%$ & $n$ & $\%$ & $n$ & $\%$ \\
\hline Caries/Restorative & 13 & 36.1 & 6 & 27.3 & 6 & 50 & 0 & 0 & 25 & 35.2 \\
\hline Fracture & 10 & 27.8 & 8 & 36.4 & 2 & 16.7 & 1 & 100 & 21 & 29.6 \\
\hline Endodontic & 9 & 25 & 5 & 22.7 & 3 & 25 & 0 & 0 & 17 & 23.9 \\
\hline Periodontal & 4 & 11.1 & 3 & 13.6 & 1 & 8.3 & 0 & 0 & 8 & 11.3 \\
\hline Total failure & 36 & 19.6 & 22 & 20 & 12 & 14.1 & 1 & 2.8 & 71 & 17.1 \\
\hline Total teeth no. & 184 & & 110 & & 85 & & 35 & & 414 & \\
\hline
\end{tabular}

TABLE 2 Failure rate stratified by causes and follow-up time
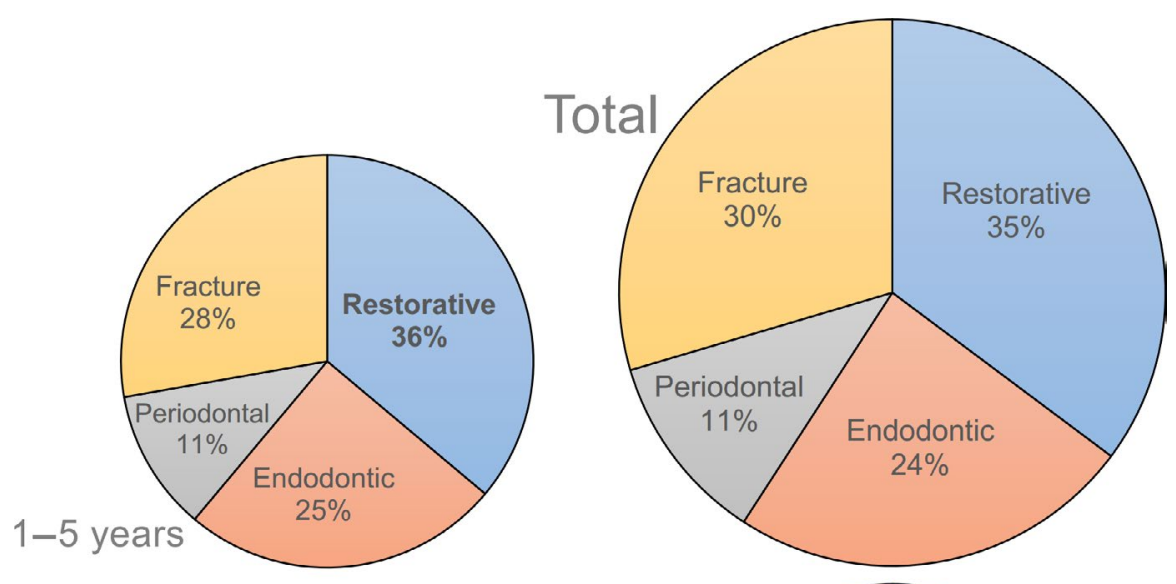

FIGURE 4 Frequency of different causes of failure and stratification by follow-up times
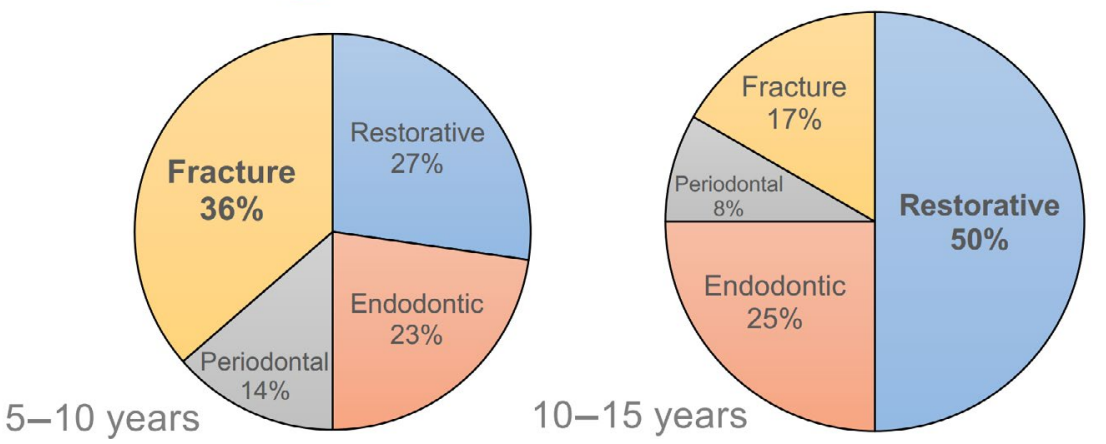

patients should be given objective information regarding both options based on the reported evidence. It is of paramount importance to explain the patient that dental implant therapy may seem predictable in replacing the tooth with an implant-supported crown, but prosthetic and biological complications may also occur in the long term. It was reported that the occurrence of peri-implantitis is around 22\% (Derks \& Tomasi, 2015). On the other hand, some limitations of CLP should be recognized, including removing excessive bone may compromise the adjacent teeth and reducing the alveolar bone that may complicate future implant placement. CLP may not be the treatment of choice for single tooth in aesthetic zone, as it may alter the gingival level compared to adjacent or contralateral tooth (Rambhia, Heshmati, Dhuru, \& lacopino, 2009). In addition, clinicians should always keep in mind that many alternative approaches are available, including subgingival restorations with tissue-friendly materials, fixed partial denture, removable partial denture or maintain the edentulous ridge. What is the best option for each patient may be different and should be discussed with the patient.

\section{5 | CONCLUSION}

Structurally compromised dentition has a reasonable 10 -year survival rate of close to $80 \%$ preserved after crown lengthening and restoration in a teaching institute. It has to be noted that these data are based on less experienced clinicians involving other limitations and patient factors attending teaching institute. Patients with high caries or fracture risk may have higher failure rate and should be managed carefully. This objective information should be available to clinicians and be presented to patients to make a fair judgement as an evidence-based and well-informed decision whether to extract or preserve the tooth. 


\section{CONFLICT OF INTEREST}

All authors disclose no conflict of interest.

\section{ORCID}

Sajjad Ashnagar (iD https://orcid.org/0000-0003-3078-8540

Shayan Barootchi iD https://orcid.org/0000-0002-5347-6577

Andrea Ravidá (iD https://orcid.org/0000-0002-3029-8130

Mustafa Tattan (iD https://orcid.org/0000-0001-7498-8064

Hom-Lay Wang (iD https://orcid.org/0000-0003-4238-1799

Chin-Wei Wang (iD https://orcid.org/0000-0001-9679-4121

\section{REFERENCES}

Allen, E. (1993). Surgical crown lengthening for function and esthetics. Dental Clinics of North America, 37(2), 163-179.

Bader, J. D., Shugars, D. A., \& Martin, J. A. (2004). Risk indicators for posterior tooth fracture. The Journal of the American Dental Association, 135(7), 883-892. https://doi.org/10.14219/jada.archive.2004.0334

Carnevale, G., Sterrantino, S., \& Di, G. F. (1983). Soft and hard tissue wound healing following tooth preparation to the alveolar crest. The International Journal of Periodontics \& Restorative Dentistry, 3(6), 36-53.

Derks, J., \& Tomasi, C. (2015). Peri-implant health and disease. A systematic review of current epidemiology. Journal of Clinical Periodontology, 42(16), S158-171.

Dibart, S., Capri, D., Kachouh, I., Dyke, T. V., \& Nunn, M. E. (2003). Crown lengthening in mandibular molars: $\mathrm{A} 5$-year retrospective radiographic analysis. Journal of Periodontology, 74(6), 815-821. https:// doi.org/10.1902/jop.2003.74.6.815

Giannobile, W., \& Lang, N. (2016). Are dental implants a panacea or should we better strive to save teeth? Journal of Dental Research, 95(1), 5-6. https://doi.org/10.1177/0022034515618942

Hempton, T. J., \& Dominici, J. T. (2010). Contemporary crown-lengthening therapy: A review. The Journal of the American Dental Association, 141(6), 647-655. https://doi.org/10.14219/jada.archive.2010.0252

Iqbal, M. K., \& Kim, S. (2008). A review of factors influencing treatment planning decisions of single-tooth implants versus preserving natural teeth with nonsurgical endodontic therapy. Journal of Endodontics, 34(5), 519-529. https://doi.org/10.1016/j.joen.2008.01.002

Krishan, R., Paqué, F., Ossareh, A., Kishen, A., Dao, T., \& Friedman, S. (2014). Impacts of conservative endodontic cavity on root canal instrumentation efficacy and resistance to fracture assessed in incisors, premolars, and molars. Journal of Endodontics, 40(8), 11601166. https://doi.org/10.1016/j.joen.2013.12.012

Lundgren, D., Rylander, H., \& Laurell, L. (2008). To save or to extract, that is the question. Natural teeth or dental implants in periodontitis-susceptible patients: clinical decision-making and treatment strategies exemplified with patient case presentations. Periodontology 2000, 47(1), 27-50.

Moghaddam, A., Radafshar, G., Taramsari, M., \& Darabi, F. (2014). Long-term survival rate of teeth receiving multidisciplinary endodontic, periodontal and prosthodontic treatments. Journal of Oral Rehabilitation, 41(3), 236-242. https://doi.org/10.1111/joor. 12136

Motohashi, M., Yamada, H., Genkai, F., Kato, H., Imai, T., Sato, S., ... Maeno, M. (2006). Employing dmft score as a risk predictor for caries development in the permanent teeth in Japanese primary school girls. Journal of Oral Science, 48(4), 233-237. https://doi.org/10.2334/ josnusd.48.233

Oakley, E., Rhyu, I.-C., Karatzas, S., Gandini-Santiago, L., Nevins, M., \& Caton, J. (1999). Formation of the biologic width following crown lengthening in nonhuman primates. International Journal of Periodontics and Restorative Dentistry, 19(6), 529-541.

Packer, G. M. (2007). The use of implants vs. endodontics: Practitioner attitudes in 2007.

Padbury, A. Jr, Eber, R., \& Wang, H. L. (2003). Interactions between the gingiva and the margin of restorations. Journal of Clinical Periodontology, 30(5), 379-385. https://doi.org/10.1034/j.1600-051X.2003.01277.x

Palomo, F., \& Kopczyk, R. A. (1978). Rationale and methods for crown lengthening. Journal of the American Dental Association, 96(2), 257-260

Patil, S. A., Kulkarni, S., Thakur, S., \& Naik, B. (2016). Crown lengthening procedure following intentional endodontic therapy for correction of supra-erupted posterior teeth: Case series with long-term followup. Journal of Indian Society of Periodontology, 20(1), 103. https://doi. org/10.4103/0972-124X.175176

Pilalas, I., Tsalikis, L., \& Tatakis, D. N. (2016). Pre-restorative crown lengthening surgery outcomes: A systematic review. Journal of Clinical Periodontology, 43(12), 1094-1108. https://doi.org/10.1111/ jcpe.12617

Rambhia, S., Heshmati, R., Dhuru, V., \& lacopino, A. (2009). Shear bond strength of orthodontic brackets bonded to provisional crown materials utilizing two different adhesives. The Angle Orthodontist, 79(4), 784-789. https://doi.org/10.2319/060908-298.1

Stockhausen, R., Aseltine, R. Jr, Matthews, J. G., \& Kaufman, B. (2011). The perceived prognosis of endodontic treatment and implant therapy among dental practitioners. Oral Surgery, Oral Medicine, Oral Pathology, Oral Radiology, and Endodontology, 111(2), e42-e47. https:// doi.org/10.1016/j.tripleo.2010.10.007

Torabinejad, M., \& Goodacre, C. J. (2006). Endodontic or dental implant therapy: The factors affecting treatment planning. The Journal of the American Dental Association, 137(7), 973-977. https://doi. org/10.14219/jada.archive.2006.0318

Torbjörner, A., \& Fransson, B. (2004). A literature review on the prosthetic treatment of structurally compromised teeth. International Journal of Prosthodontics, 17(3), 369-376.

How to cite this article: Ashnagar S, Barootchi S, Ravidá A, Tattan M, Wang H-L, Wang C-W. Long-term survival of structurally compromised tooth preserved with crown lengthening procedure and restorative treatment: A pilot retrospective analysis. J Clin Periodontol. 2019;46:751-757. https://doi.org/10.1111/jcpe.13124 